\title{
Cerebral Blood Flow Improvement after Indirect Revascularization for Pediatric Moyamoya Disease: A Statistical Analysis of Arterial Spin-Labeling MRI
}

\author{
(D). Blauwblomme, (D). Lemaitre, (D). Naggara, (D) R. Calmon, (D). Kossorotoff, (D) M. Bourgeois, (D). Mathon, (D) S. Puget, \\ (D) M. Zerah, (DF. Brunelle, (D). Sainte-Rose, and (D) N. Boddaert
}

\begin{abstract}
BACKGROUND AND PURPOSE: The severity of Moyamoya disease is generally scaled with conventional angiography and nuclear medicine. Arterial spin-labeling MR imaging is now acknowledged for the noninvasive quantification of cerebral blood flow. This study aimed to analyze CBF modifications with statistical parametric mapping of arterial spin-labeling MR imaging in children undergoing an operation for Moyamoya disease.
\end{abstract}

MATERIALS AND METHODS: We included 15 children treated by indirect cerebral revascularization with multiple burr-holes between 2011 and 2013. Arterial spin-labeling MR imaging and T1 sequences were then analyzed under SPM8, according to the general linear model, before and after the operation ( 3 and 12 months). Voxel-based analysis was performed at the group level, comparing all diseased hemispheres with all normal hemispheres and, at the individual level, comparing each patient with a control group.

RESULTS: Group analysis showed statistically significant preoperative hypoperfusion in the MCA territory in the Moyamoya hemispheres and a significant increase of cerebral perfusion in the same territory after revascularization $(P<.05$ family-wise errorcorrected). Before the operation, individual analysis showed significant hypoperfusion for each patient co-localized with the angiographic defect on DSA. All except 1 patient had improvement of CBF after revascularization, correlated with their clinical status.

CONCLUSIONS: SPM analysis of arterial spin-labeling MR imaging offers a noninvasive evaluation of preoperative cerebral hemodynamic impairment and an objective assessment of postoperative improvement in children with Moyamoya disease.

ABBREVIATIONS: $A S L=$ arterial spin-labeling; $M M=$ pediatric Moyamoya disease; $S P M=$ statistical parametric mapping

$\mathbf{M}_{\mathrm{p}}^{\circ}$ oyamoya disease (MM) is a vascular disease defined by a progressive occlusion of the supraclinoidal internal carotid arteries along with the development of leptomeningeal collaterals. ${ }^{1}$ Its natural history includes occurrence of transient ischemic attacks, ischemic strokes, or intracerebral hemorrhage. Because there is currently no efficient medical treatment, cerebral revas-

Received June 27, 2015; accepted after revision August 20.

From the Université René Descartes (T.B., O.N., R.C., B.M., S.P., M.Z., F.B., C.S.-R., N.B.), PRES Sorbonne Paris Cité, Paris, France; APHP, Departments of Pediatric Neurosurgery (T.B., M.B., S.P., M.Z., C.S.-R.) and Neuroradiology (O.N., R.C., F.B., N.B.), Hospital Necker, Paris, France; French Institute of Health and Medical Research U1000 (T.B., H.L., R.C., N.B.), Institut Imagine, University Paris-Sud 11 and University Paris Descartes, Paris, France; Department of Neuroradiology (O.N.), French Institute of Health and Medical Research U894, Hospital Sainte-Anne, Paris, France; APHP, Department of Pediatric Neurology (M.K.), French Center for Pediatric Stroke, Hospital Necker, Paris, France; and UMR 1163 (N.B.), Institut Imagine, Paris, France.

Please address correspondence to Thomas Blauwblomme, MD, Service de Neurochirurgie Pédiatrique. Hôpital Necker, 149 rue de Sèvres, 75743 Paris Cedex 15,

France; e-mail: thomas.blauwblomme@nck.aphp.fr

http://dx.doi.org/10.3174/ajnr.A4592 cularization is the sole option when cerebral hemodynamics are compromised. Currently, morphologic MR imaging is sufficient for the diagnosis of MM because it shows arterial stenosis and its consequences on the brain (ischemic strokes, ivy sign). ${ }^{2,3}$ However, selection of surgical candidates requires a grading of MM severity with a measure of the cerebral blood flow. Several imaging techniques are available, such as xenon-enhanced CT or DSC-weighted MR imaging; however, nuclear medicine with $\mathrm{H}_{2}\left[{ }^{15} 0\right]$-PET and ${ }^{123}$ I-iodoamphetamine SPECT studies is still the criterion standard to quantify alteration of CBF and cerebrovascular reserve. ${ }^{4}$

Recently, to avoid radiation exposure, arterial spin-labeling (ASL) MR imaging has been successfully developed to quantify CBF alterations in different stroke conditions, including MM., This MR imaging has the advantage of being noninvasive because it uses water protons as an endogenous tracer to measure CBF, rather than intravenous injection of a contrast agent, which may be a concern in the pediatric population. Because children under- 


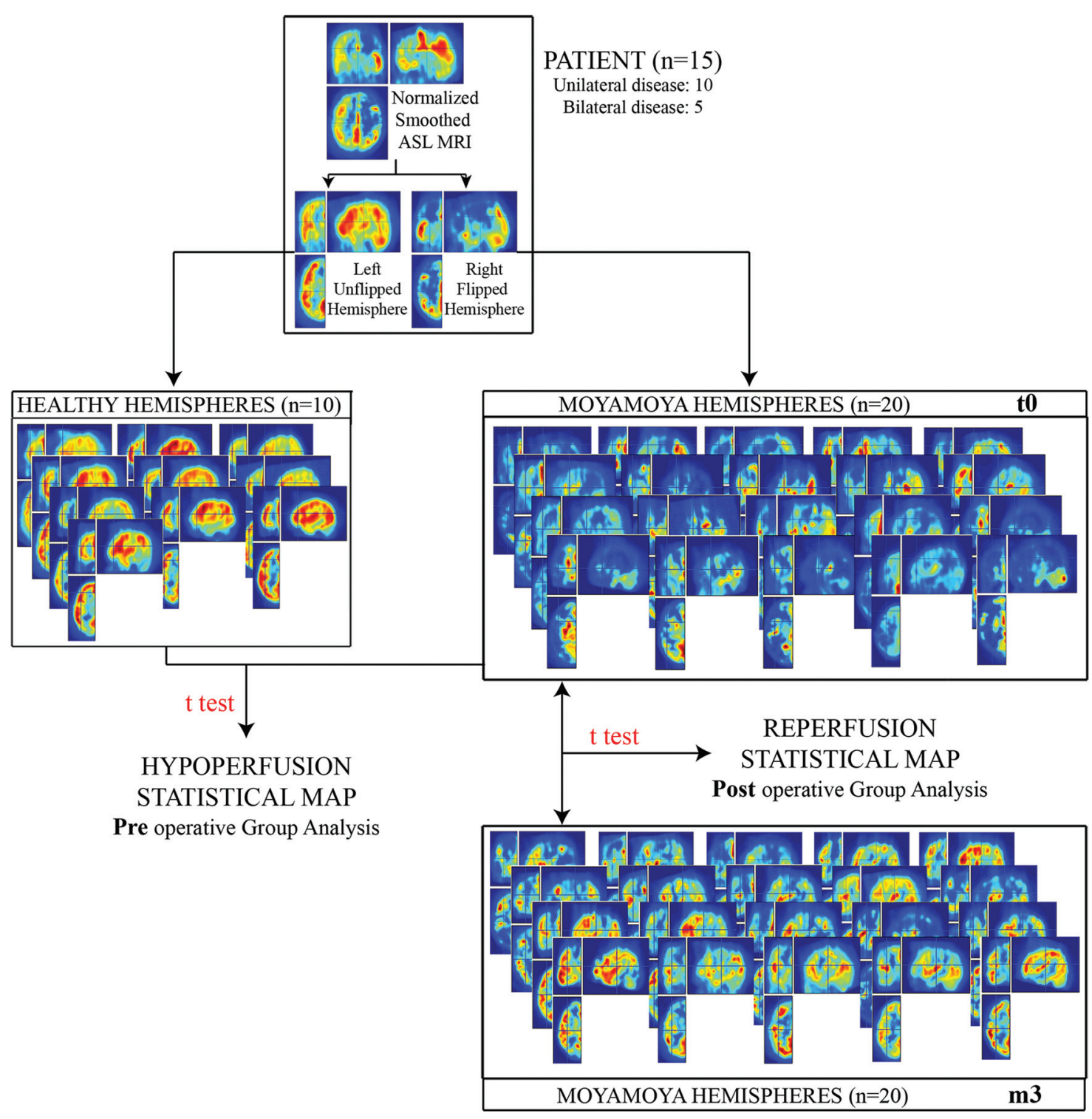

FIG 1. Methodology of the voxel-based ASL MR imaging analysis at the group level. For each of the 15 patients, smoothed normalized MRIs were split into 2 distinct hemispheres. The right hemispheres are flipped along the midsagittal plane. Five patients had bilateral Moyamoya disease, whereas 10 patients had unilateral disease; therefore there are 20 hemispheres in the Moyamoya hemisphere group and 10 hemispheres in the healthy group. Preoperative hypoperfusion maps were generated while comparing non-Moyamoya with Moyamoya hemispheres with a voxel-based analysis according to the general linear model. Postoperative reperfusion maps were obtained with intrinsic comparison of the Moyamoya hemisphere group 3 months after the operation with the preoperative ASL MR imaging, according to the general linear model.

going an operation for MM need repeated quantification of their CBF before and after surgery, ASL MR imaging may be of particular interest, and statistical approaches, as described with statistical parametric analysis (SPM) of ASL MR imaging in cognitive series, may allow intraindividual and group analysis of CBF comparisons before and after the operation. ${ }^{7}$ This could allow a noninvasive follow-up of patients having undergone an operation and help to prevent recurrent stroke in patients with persistent hypoperfusion.

The aim of the present study was to analyze the modifications of cerebral blood flow in a European cohort of 15 children with Moyamoya disease consecutively treated with indirect cerebral revascularization, by using SPM analysis at the individual and group levels, with pseudocontinuous ASL MR imaging.

\section{MATERIALS AND METHODS \\ Study Design}

We performed a monocentric retrospective analysis of all patients having undergone an operation for a MM between 2011 and 2013. A preoperative MR imaging was performed the week before the operation, and $2 \mathrm{MR}$ imaging examinations were performed according to the same protocol 3 months and 1 year after the operation, respectively.

\section{Patients}

Indications for cerebral revascularization were decided in a multidisciplinary meeting according to clinical, angiographic, and MR imaging data. PET and SPECT studies were not performed because they were not available in our hospital.

All patients having undergone an operation between 2011 and 


\section{PATIENT (n=1)}

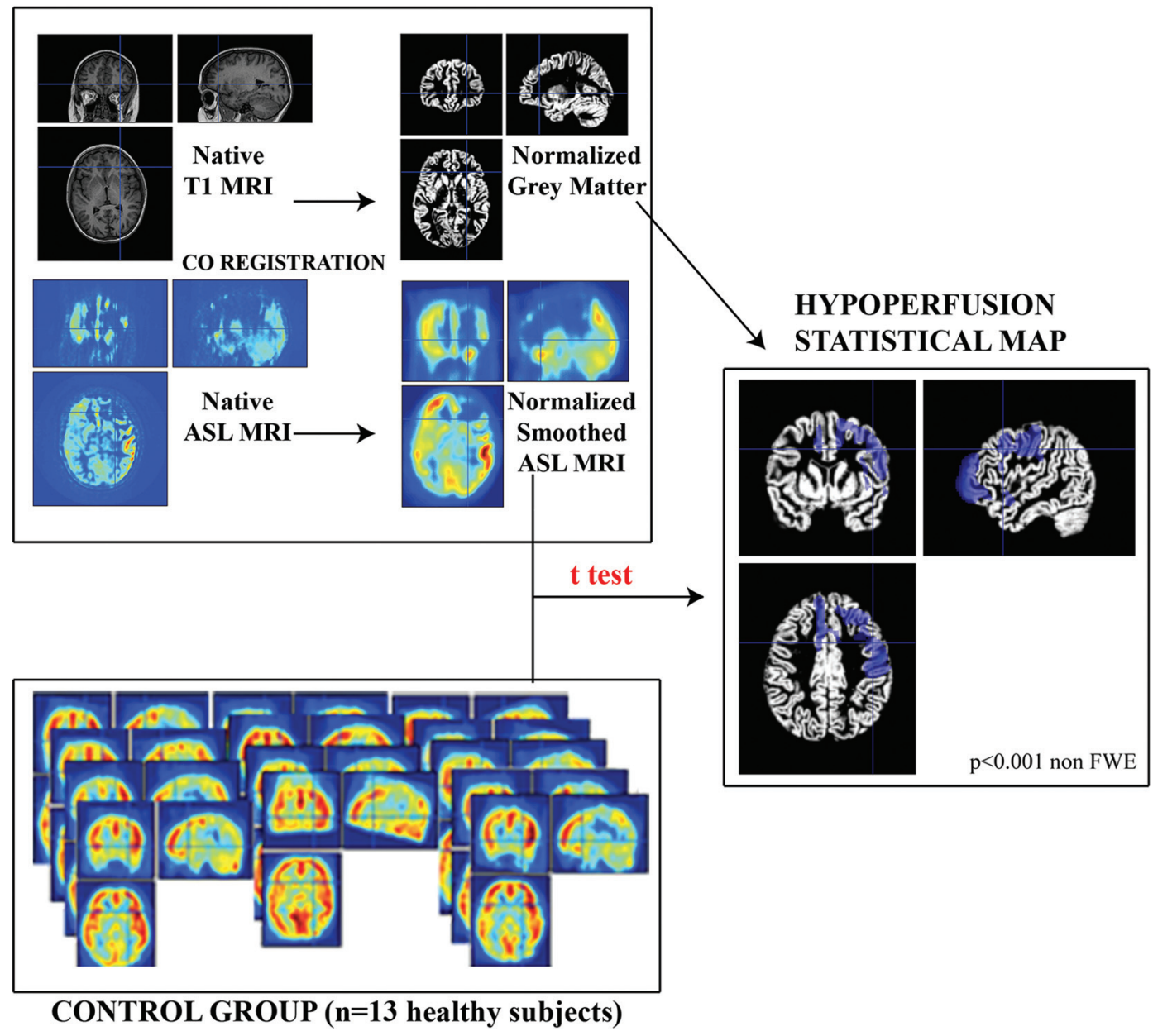

FIG 2. Methodology of the voxel-based ASL MR imaging analysis at the individual level. For each patient, ASL MR imaging was coregistered with the TI MR imaging and then smoothed and normalized under SPM8. A voxel-based analysis was then performed between the patients with ASL MR imaging and the group of 13 healthy children, with the general linear model under SPM8. Areas with significant hypoperfusion were then displayed on the normalized gray matter (blue areas).

2013 were included in the study. Postoperative outcome was assessed by a pediatric neurologist in regard to postoperative ischemic events (TIA and strokes).

We used a control group with 13 subjects comparable for age (mean age, $6.8 \pm 2.8$ years; range, 3-11 years) and sex (male/ female ratio $=0.75$ ). Their anatomic brain MR imaging findings were normal, and indications for imaging were the following: systemic disease $(n=5)$, mild psychiatric disorder $(n=3)$, ophthalmologic disorder $(n=3)$, and headaches $(n=2)$. None had neurologic or neurosurgical disorders.

\section{Surgical Technique}

All patients underwent the operation with the same indirect cerebral revascularization technique: multiple burr-hole surgery. ${ }^{8}$ Briefly, a uni- or bilateral coronal incision with subgaleal dissection exposes the skull vault. Between 15 and 20 burr-holes are drilled according to the following technique: A triangular periosteal flap is elevated, a $<1 \mathrm{~cm}$ burr-hole is drilled, and the dura and arachnoid layers are opened. The flap is then inserted in the subdural space; and 2 layers of watertight closure of the skin are made, with or without drainage. Progressive spontaneous anastomoses then occur between pial vessels and external carotid branches during the weeks following the operation.

\section{MR Imaging Sequences}

MR images were obtained on a 1.5T Signa HDxt system (GE Healthcare, Milwaukee, Wisconsin) by using a 12-channel headneck-spine coil. Brain MR imaging protocol included at least a 3D T1-weighted fast-spoiled gradient-recalled sequence (TR/TE, 16.4/7.2 ms; flip angle, $13^{\circ}$; matrix size, $512 \times 512$; FOV, $22 \times 22$ $\mathrm{cm}$, with 228 axial sections at a thickness of $0.6 \mathrm{~mm}$ ) and $3 \mathrm{D}$ time-of-flight, axial FLAIR, axial T2-weighted, and diffusion and noncontrast perfusion imaging with 3D pseudocontinuous ASL MR imaging (40 axial partitions of $4 \mathrm{~mm}$ thickness; FOV, $240 \times$ $240 \mathrm{~mm}$; acquisition matrix, 8 spiral arms in each 3D partition; TE, 10.5 ms; TR, $4428 \mathrm{~ms}$; postlabeling delay, $1025 \mathrm{~ms}$; flip angle, $155^{\circ}$; acquisition time, 4 minutes 17 seconds). No injection was required.

MR images were acquired before the operation ( $\mathrm{t} 0$ ) and $3(\mathrm{~m} 3)$ and 12 months (m12) after the operation without general anesthesia. Sleep could be induced by premedication when needed $(0.2 \mathrm{mg} / \mathrm{kg}$ of sodium pentobarbital) before 6 years of age. 

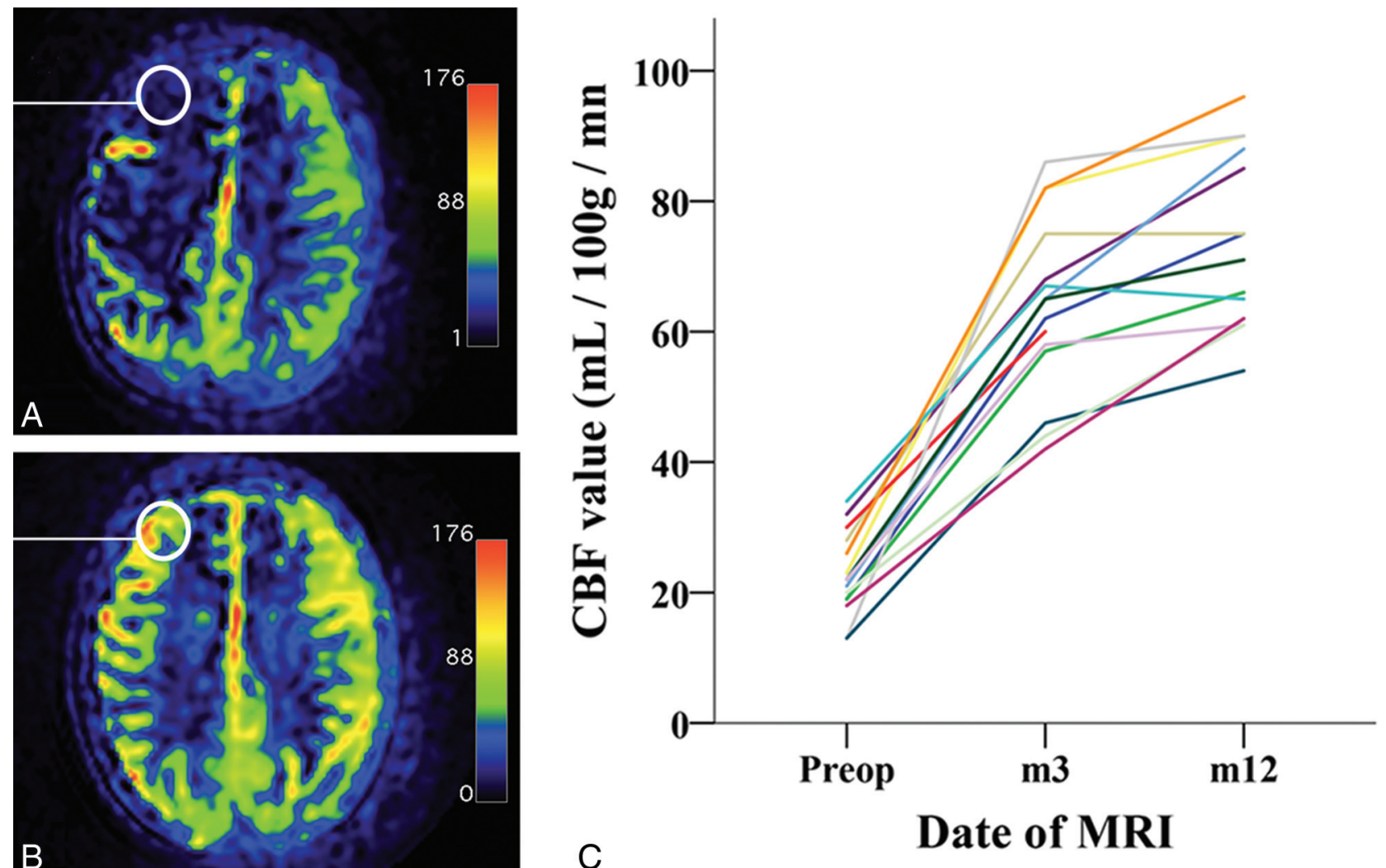

$\mathrm{B}$

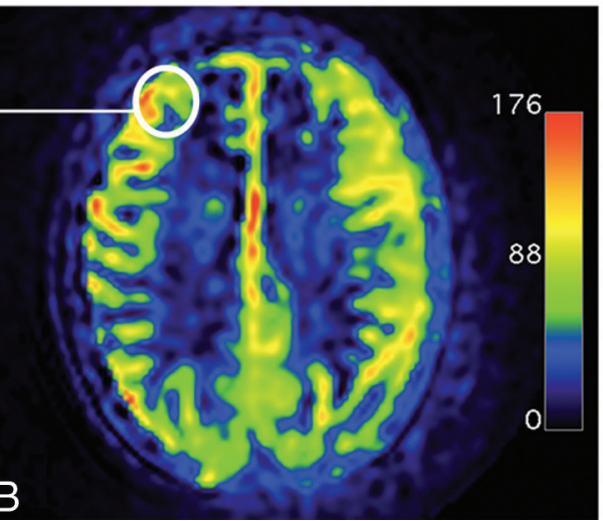

$\mathrm{C}$

FIG 3. Quantitative analysis of ASL MR imaging. A, Preoperative ASL MR imaging. Note hypoperfusion on the right frontal lobe and central region. The color scale unit of the CBF map is $\mathrm{mL} / 100 \mathrm{mg} / \mathrm{min}$. B. Postoperative ASL MR imaging (12 months after the operation) of the same patient. Note increased $\mathrm{CBF}$, with normal values of $\mathrm{CBF}$ in the frontal lobe and central area. C, Diagram shows the evolution of CBF values in an ROI in the frontal lobe (white circle on $A$ and $B$ ) for each patient before the operation and 3 and 12 months after the operation. Preop indicates preoperative.

\section{Statistical Methods: Voxel-Based Analysis of ASL MR Imaging}

MR images were preprocessed by using statistical parametric mapping (SPM8 software; www.fil.ion.ucl.ac.uk/spm/software/ spm8), implemented in Matlab (MathWorks, Natick, Massachusetts) and analyzed by using a voxel-based approach. Native T1weighted images were segmented into gray matter, white matter, and CSF by using the VBM8 segmentation toolbox (http:// www.neuro.uni-jena.de/vbm/). ${ }^{9}$ The ASL images were coregistered to the corresponding native gray images and spatially normalized to the Montreal Neurological Institute space by using the deformation matrices from the segmentation process. The resulting ASL images were smoothed by using an isotropic Gaussian filter of $10 \mathrm{~mm}$. To compare Moyamoya and non-Moyamoya hemispheres, we generated mirror images by flipping each smoothed ASL image about the sagittal plane through the midline. Only the left unflipped hemisphere and the right flipped hemisphere were considered within the statistical analyses. Voxelbased group analyses (Fig 1) were performed within the framework of the general linear model by using a flexible factorial design considering 3 factors: subject, time ( $\mathrm{t} 0, \mathrm{~m} 3, \mathrm{~m} 12)$, and hemisphere status (healthy versus Moyamoya disease). Sex, etiology, relapse, and types of symptoms were included as confounding covariates. A proportional scaling of the ASL data was applied and set to a grand mean scaled value of $50 \mathrm{~mL} / \mathrm{dL} / \mathrm{min}$ to minimize intersubject variability.

Eventually, we performed individual analyses. We used a con- trol group of 13 subjects matched with our patient group for sex and age. For each patient, we performed a voxel-based comparison between the control group and the patient ASL images preoperatively and at last follow-up (Fig 2).

The significance level was set to $P=.05$ corrected, family-wise error-corrected for multiple comparisons at the voxel level with a masking threshold set to $70 \mathrm{~mL} / \mathrm{dL} / \mathrm{min}$.

\section{Statistical Methods: Quantitative Analysis of ASL MR Imaging}

A 1-cm ROI was also chosen in the frontal lobe (Fig 3), where GE software quantified CBF, before and after the operation. The ROI was chosen at the level of the corpus callosum, in the anterior third of the dorsolateral frontal lobe cortex because this region displayed important variations of CBF on the SPM analysis at the group and the individual levels. Moreover, this area in the MCA territory is particularly affected in Moyamoya vasculopathy.

Statistical analysis then compared preoperative and postoperative values, in the Moyamoya hemispheres and in the healthy hemispheres, with the Friedman nonparametric test.

\section{RESULTS}

\section{Patients}

Fifteen children were included in the study. A summary of the patient characteristics is shown in Table 1. Bilateral revascularization was performed in 5 patients, and unilateral revascularization, 
Table 1: Summary of patient characteristics

\begin{tabular}{|c|c|}
\hline Characteristic & \\
\hline $\begin{array}{l}\text { Median age at operation } \\
\text { Range }\end{array}$ & $\begin{array}{l}6.8 \pm 3.4 y \\
2.7-14 y\end{array}$ \\
\hline \multicolumn{2}{|l|}{ Sex } \\
\hline 10 Males/5 females & $M / F$ ratio $=2$ \\
\hline \multicolumn{2}{|l|}{ Ethnic origin } \\
\hline Caucasian & 11 \\
\hline North African & 2 \\
\hline Sub-Saharan African & 1 \\
\hline Asian & 1 \\
\hline \multicolumn{2}{|l|}{ Etiology } \\
\hline Disease & 8 \\
\hline NF-1 & 5 \\
\hline Down syndrome & 1 \\
\hline CBL gene mutation & 1 \\
\hline \multicolumn{2}{|l|}{ Modality of revelation } \\
\hline Ischemic stroke & 5 \\
\hline TIA & 3 \\
\hline MRI follow-up NF-1 & 3 \\
\hline $\mathrm{ICH}$ & 3 \\
\hline Headaches & 1 \\
\hline \multicolumn{2}{|l|}{ Clinical symptoms } \\
\hline Neurologic deficits & 6 \\
\hline Partial & 4 \\
\hline Severe & 2 \\
\hline Headaches & 6 \\
\hline Epilepsy & 3 \\
\hline No symptoms & 3 \\
\hline \multicolumn{2}{|l|}{ Preoperative stroke on MRI } \\
\hline No stroke & 6 \\
\hline Single territory & 5 \\
\hline Multiple territory & 1 \\
\hline $\mathrm{ICH} / \mathrm{IVH}$ & 3 \\
\hline \multicolumn{2}{|l|}{ Surgery (multiple burr-holes) } \\
\hline Bilateral & 5 \\
\hline Unilateral right & 6 \\
\hline Unilateral left & 4 \\
\hline \multicolumn{2}{|l|}{ Postoperative outcome } \\
\hline Follow-up & $28 \pm 7 \mathrm{mo}$ \\
\hline Recurrent TIAs & 1 \\
\hline Second surgery needed & 1 \\
\hline
\end{tabular}

Note:-NF-1 indicates neurofibromatosis type 1; ICH, intracerebral hematoma; $\mathrm{CBL}$, casitas B lineage lymphomas; IVH, intraventricular hemorrhage.

in 10 patients. Recurrence of TIA in 1 patient required a second revascularization procedure.

\section{ASL MR Imaging: Direct Quantitative Analysis}

In the hemispheres affected by Moyamoya vasculopathy, the mean preoperative CBF in the frontal lobe was $22 \pm 6.3 \mathrm{~mL} /$ $100 \mathrm{mg} / \mathrm{min}$ (range, $13-34 \mathrm{~mL} / 100 \mathrm{mg} / \mathrm{min}$ ), whereas it was $65 \pm$ $13.6 \mathrm{~mL} / 100 \mathrm{mg} / \mathrm{min}$ (range, $42-86 \mathrm{~mL} / 100 \mathrm{mg} / \mathrm{min}$ ) and $73 \pm$ $13.4 \mathrm{~mL} / 100 \mathrm{mg} / \mathrm{min}$ (range, $54-96 \mathrm{~mL} / 100 \mathrm{mg} / \mathrm{min}$ ) 3 and 12 months after the operation, respectively (Fig 3 and Table 2). This increase was statistically significant (Friedman nonparametric test, $P<.001)$.

On the other hand, in the non-Moyamoya hemispheres, mean preoperative $\mathrm{CBF}$ in the frontal lobe was $80.8 \pm 11.3$ $\mathrm{mL} / 100 \mathrm{mg} / \mathrm{min}$ (range, $64-93 \mathrm{~mL} / 100 \mathrm{mg} / \mathrm{min}$ ), and there was no statistically significant change postoperatively (Friedman test, $P=.703$ ) after 3 months (mean CBF, $83 \pm 10.8$ $\mathrm{mL} / 100 \mathrm{mg} / \mathrm{min}$; range, $61-94 \mathrm{~mL} / 100 \mathrm{mg} / \mathrm{min}$ ) and 12 months (mean CBF, $83.2 \pm 10.7 \mathrm{~mL} / 100 \mathrm{mg} / \mathrm{min}$; range, 62-93 $\mathrm{mL} / 100 \mathrm{mg} / \mathrm{min})$.

\section{ASL MR Imaging: Group Analysis}

Comparison of MM hemispheres $(n=20)$ with non-MM hemispheres $(n=10)$ at t0 showed a preoperative significant decrease of CBF $(P<.05$ family-wise error-corrected) located in the MCA territory (Fig 4).

Intrinsic comparison of the MM hemispheres between t0 and follow-up showed a significant increase of the CBF $(P<.05$ family-wise error-corrected $)$ within the frontal lobe and temporoparietal junction at 3 months (m3) and 12 months (m12) after the operation. Conversely, intrinsic comparisons of the healthy hemisphere at $\mathrm{m} 3$ and $\mathrm{m} 12$ with the preoperative period did not show any significant modification of the CBF.

\section{ASL MR Imaging: Individual Analysis}

Preoperative comparisons of individual patients with $\mathrm{MM}$ with the control group showed, in all the cases, hypoperfusion $(P<.05$ family-wise error-corrected) in the MCA territory (Fig 5). Postoperative comparison of individual patients with $\mathrm{MM}$ with the control group showed dramatic improvement of cerebral perfusion in the operated territory. This was correlated with a good clinical outcome (no postoperative stroke or TIA) in all except 1 patient.

Most interesting, this latter patient had recurrent TIA, and statistical analysis showed persistent hypoperfusion, co-localized within an area where no burr-holes had been drilled, allowing a further targeted second-stage operation.

In another patient with good clinical results, contralateral asymptomatic hypoperfusion in the anterior cerebral artery territories was detected.

\section{DISCUSSION}

We report a prospective series of 15 children undergoing an operation for a Moyamoya disease with indirect cerebral revascularization followed by pre- and postoperative ( 3 months and 1 year after the operation) ASL MR imaging. Statistical parametric mapping analysis of ASL MR imaging showed, at the group level, preoperative statistical hypoperfusion in the MCA territory and a postoperative increase of $\mathrm{CBF}$ in the same territory after the operation. Individual analysis displayed, in all cases, territories of statistically significant hypoperfusion in the MCA territory and a postoperative improvement in all except 1 case.

The present study has some biases. We did not evaluate the cerebrovascular reserve, and ASL MR imaging was not compared with other reference imaging like PET, SPECT, perfusion CT, or DSC MR imaging because these techniques were not performed in our center.

In the present study, we provide more evidence to support pseudocontinuous ASL MR imaging in the initial cerebral perfusion assessment and follow-up of MM. This technique does not require contrast injection and allows quantification of the cerebral blood flow and cerebrovascular reserve (if acetazolamide studies are performed). Its first application in MM was reported in 2011, with the demonstration of good agreement between ASL MR imaging and DSA, in showing spontaneous transosseous collaterality. In this study, ASL sensitivity and specificity were 0.83 and 0.82 , respectively. ${ }^{5}$ Further studies, mainly from Ja- 
Table 2: Quantitative CBF values from the frontal lobe ROI ${ }^{\mathrm{a}}$

\begin{tabular}{|c|c|c|c|c|c|c|c|}
\hline \multirow[b]{2}{*}{ Patient } & \multirow{2}{*}{$\begin{array}{l}\text { Side of the } \\
\text { Disease }\end{array}$} & \multicolumn{3}{|c|}{$\begin{array}{l}\text { Healthy Hemisphere CBF } \\
\text { Value }(\mathrm{mL} / 100 \mathrm{mg} / \mathrm{min})\end{array}$} & \multicolumn{3}{|c|}{$\begin{array}{l}\text { Diseased Hemisphere CBF } \\
\text { Value (mL/100 mg/min) }\end{array}$} \\
\hline & & Preop & M3 & M12 & Preop & M3 & M12 \\
\hline 1 & Bilateral & NA & NA & NA & 19 & 62 & 75 \\
\hline 2 & Unilateral right & 64 & 71 & 62 & 19 & 57 & 66 \\
\hline 3 & Unilateral left & 93 & 94 & 91 & 28 & 75 & 75 \\
\hline 4 & Unilateral left & 78 & 86 & 88 & 32 & 68 & 85 \\
\hline 5 & Bilateral & NA & NA & NA & 23 & 82 & 90 \\
\hline 6 & Bilateral & NA & NA & NA & 30 & 60 & \\
\hline 7 & Bilateral & NA & NA & NA & 34 & 67 & 65 \\
\hline 8 & Unilateral right & 78 & 81 & 81 & 13 & 86 & 90 \\
\hline 9 & Unilateral right & 79 & 90 & 90 & 21 & 65 & 88 \\
\hline 10 & Unilateral right & 92 & 90 & 93 & 22 & 65 & 71 \\
\hline 11 & Unilateral right & 91 & 82 & 88 & 26 & 82 & 96 \\
\hline 12 & Bilateral & NA & NA & NA & 22 & 58 & 61 \\
\hline 13 & Bilateral & NA & NA & NA & 13 & 46 & 54 \\
\hline 14 & Unilateral left & 64 & 61 & 69 & 20 & 44 & 61 \\
\hline 15 & Unilateral right & 89 & 92 & 87 & 18 & 42 & 62 \\
\hline
\end{tabular}

Note:-NA indicates no available data because there was no healthy hemisphere; Preop, preoperative.

${ }^{a}$ In case of bilateral disease, an ROI in the right hemisphere was chosen.

\section{to PRE OPERATIVE HYPOPERFUSION MAP}
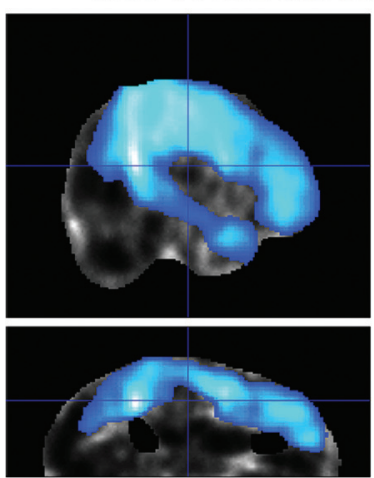
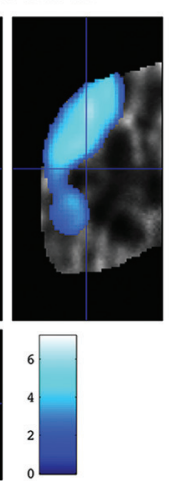
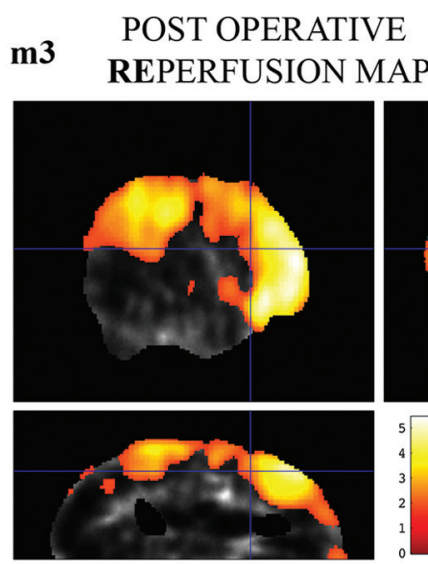
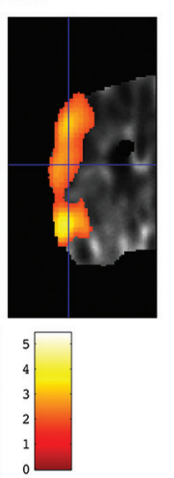

m12 POST OPERATIVE

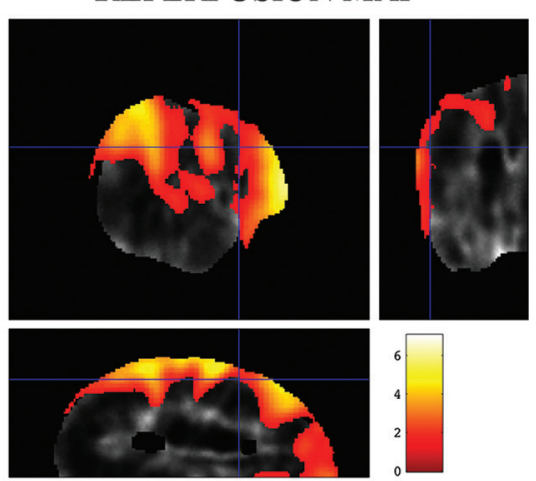

FIG 4. Results of SPM analysis of cerebral perfusion at the group level. The significance level is set at $P<.05$. Color bar displays the $z$ score. T0: Comparison of Moyamoya hemispheres with healthy hemispheres before the operation. The blue areas represent cerebral areas with statistically significant hypoperfusion in the Moyamoya hemisphere group. M3: Comparison of Moyamoya hemispheres 3 months after the operation with Moyamoya hemispheres before the operation. The yellow and red area shows cerebral areas with a significant increase in cerebral blood flow 3 months after revascularization. M12: Comparison of Moyamoya hemispheres 12 months after the operation with Moyamoya hemispheres before the operation. The yellow and red area shows cerebral areas with a significant increase in cerebral blood flow 12 months after multiple burr-hole operations. There is an increase of the $z$ score and the size of the revascularized area.

pan, focused on the ability of ASL-MR imaging to quantify decreased CBF. Quantitative analysis of ASL MR imaging and comparison with the values measured with ${ }^{123}$ I-iodoamphetamine SPECT in patients with MM showed that ASL MR imaging could identify a decrease of CBF, which was of less amplitude than the decrease measured with SPECT studies. ${ }^{10}$ The correlation between $\mathrm{CBF}$ values and cerebrovascular reserve measured with SPECT studies in MM was good $(r=0.80)^{11,12}$ and comparable with studies addressing carotid stenosis from other etiologies $(r=0.92) .{ }^{13}$

Most interesting, ASL values were adversely affected by the degree of steno-occlusive changes assessed by MRA on the carotid bifurcation. ${ }^{14}$ Comparisons of CBF measured with ASL MR imaging in MM were also performed with $\mathrm{H}_{2}\left[{ }^{15} 0\right]$-PET studies and dynamic susceptibility contrast MR imaging, with an excellent correlation ( $r=0.79$ and 0.67 , respectively). ${ }^{6,15}$ Excellent correlations between arterial transit time and cerebral blood flow val- ues were also found between perfusion $\mathrm{CT}$ and pseudocontinuous ASL MR imaging. ${ }^{16}$

In the present study, quantitative analysis of $\mathrm{CBF}$ in the frontal lobe showed postoperative improvement in the Moyamoya hemispheres and not in the healthy hemispheres. However, selection of an ROI introduces some bias because it depends on the observer's choice and limits the spatial sampling of the cerebral cortex. Therefore, we also used another methodology to analyze CBF with ASL MR imaging, by analyzing variation with the norm (healthy hemispheres or control subjects) rather than quantifying absolute $\mathrm{CBF}$ values. To minimize intersubject variations of $\mathrm{CBF}$ and to increase the sensitivity of this imaging technique, we used whole-brain normalization and voxel-based analysis of ASL MR imaging, as previously described in cognitive studies. ${ }^{7}$ At the group level, we were able to display preoperative hypoperfusion in the MCA territory and a postoperative increase of CBF perfusion, therefore validating the surgical technique. At the individual level, 

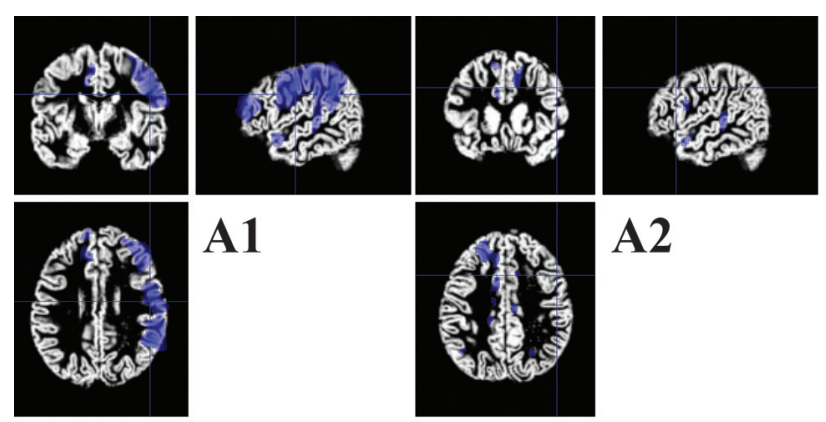

A1

A1

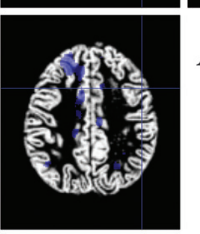

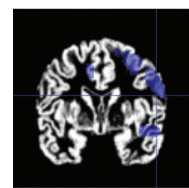
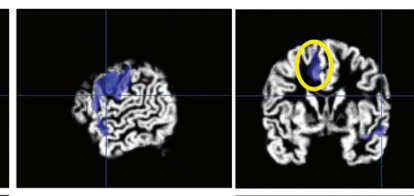

B1
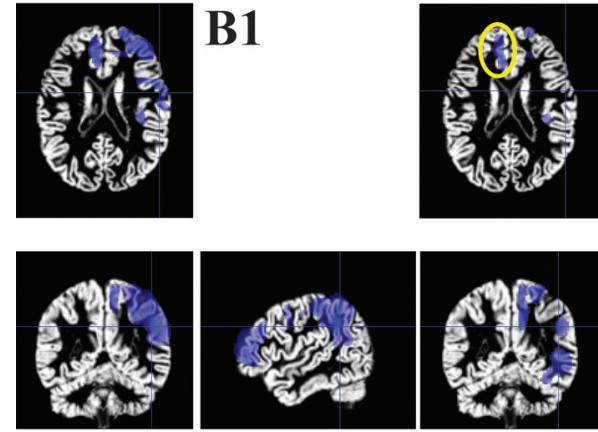

C1
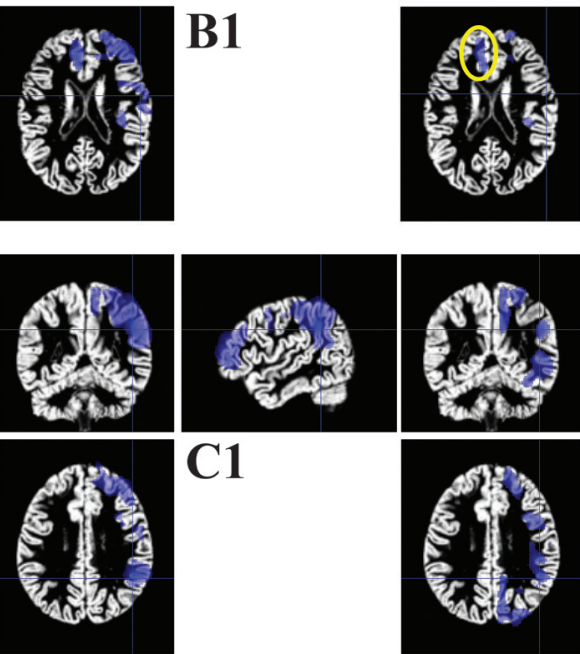

A2

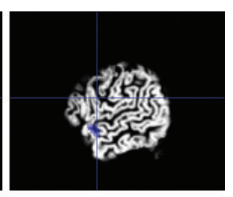

B2

FIG 5. SPM analysis of cerebral perfusion at the individual level for 3 distinct patients. We used each patient's gray matter map obtained with the VBM toolbox as a template. The blue areas display voxels of significant decrease of $\mathrm{CBF}$ values compared with the control group $(P<.001$ non-family-wise error). A1: Right unilateral hypoperfusion in the middle cerebral artery territory. A2: Postoperative CBF map shows excellent reperfusion 12 months after the operation. B1: Right hypoperfusion in the territory of the middle cerebral artery and left hypoperfusion in the territory of the left anterior cerebral artery. B2: Postoperative control shows a good result on the right hemisphere but residual hypoperfusion on the left side (the patient had undergone a unilateral right surgical procedure 12 months before). Cl: Right unilateral hypoperfusion in the middle cerebral artery territory. C2: Postoperative control shows failure of the revascularization procedure; at 6 months after the operation, the patients had recurrent TIAs and wide areas remained statistically hypoperfused in the territory of the MCA. The 3D scan shows lack of a burr-hole in the posterior temporoparietal junction.

SPM analysis showed objective areas of hypoperfusion compared with a control group. It could, therefore, be used as a screening imaging test in asymptomatic patients, at risk for $\mathrm{MM}$, as in type 1 neurofibromatosis or sickle cell disease. It allows postoperative noninvasive follow-up of the patients and an evaluation of the success or failure of revascularization, thus permitting further targeted surgeries. ASL MR imaging could, therefore, be of particular interest to initiate treatment and prevent stroke occurrence preoperatively but also postoperatively in case of residual hypoperfusion.

\section{CONCLUSIONS}

SPM analysis of ASL MR imaging in pediatric Moyamoya disease allows, at the group level, an evaluation of the surgical technique of revascularization. At the individual level, ASL MR imaging offers a noninvasive evaluation of initial hemodynamic impairment and objective assessment of postoperative improvement. In addition, ASL MR imaging may help in the decision to retreat in case of recurrent stroke or TIA.

\section{REFERENCES}

1. Scott RM, Smith ER. Moyamoya disease and Moyamoya syndrome. N Engl J Med 2009;360:1226-37 CrossRef Medline

2. Houkin K, Nakayama N, Kuroda S, et al. Novel magnetic resonance angiography stage grading for Moyamoya disease. Cerebrovasc Dis 2005;20:347-54 Medline

3. Fujiwara H, Momoshima S, Kuribayashi S. Leptomeningeal high signal intensity (ivy sign) on fluid-attenuated inversion-recovery (FLAIR) MR images in Moyamoya disease. Eur J Radiol 2005;55: 224-30 CrossRef Medline

4. Lee M, Zaharchuk G, Guzman R, et al. Quantitative hemodynamic studies in Moyamoya disease. Neurosurg Focus 2009;26:E5 CrossRef Medline

5. Zaharchuk G, Do HM, Marks MP, et al. Arterial spin-labeling MRI can identify the presence and intensity of collateral perfusion in patients with Moyamoya disease. Stroke 2011;42:2485-91 CrossRef Medline

6. Goetti R, Warnock G, Kuhn FP, et al. Quantitative cerebral perfusion imaging in children and young adults with Moyamoya disease: comparison of arterial spin-labeling-MRI and H(2)[(15)O]-PET. AJNR Am J Neuroradiol 2014;35:1022-28 CrossRef Medline

7. Aslan S, Lu H. On the sensitivity of ASL MRI in detecting regional differences in cerebral blood flow. Magn Reson Imaging 2010;28: 928-35 CrossRef Medline

8. Sainte-Rose C, Oliveira R, Puget S, et al. Multiple bur hole surgery for the treatment of Moyamoya disease in children. J Neurosurg 2006:105(6 suppl):437-43 Medline

9. Luders E, Gaser C, Jancke L, et al. A voxel-based approach to gray matter asymmetries. Neuroimage 2004;22:656-64 Medline

10. Noguchi T, Kawashima M, Irie H, et al. Arterial spin-labeling MR imaging in Moyamoya disease compared with SPECT imaging. Eur J Radiol 2011;80:e557-62 CrossRef Medline

11. Sugino T, Mikami T, Miyata K, et al. Arterial spin-labeling magnetic resonance imaging after revascularization of Moyamoya disease. $J$ Stroke Cerebrovasc Dis 2013;22:811-16 CrossRef Medline

12. Noguchi T, Kawashima M, Nishihara M, et al. Noninvasive method for mapping CVR in Moyamoya disease using ASL-MRI. Eur J Radiol 2015;84:1137-43 CrossRef Medline

13. Uchihashi Y, Hosoda K, Zimine I, et al. Clinical application of arterial spin-labeling MR imaging in patients with carotid stenosis: quantitative comparative study with single-photon emission CT. AJNR Am J Neuroradiol 2011;32:1545-51 CrossRef Medline

14. Noguchi T, Kawashima M, Nishihara M, et al. Arterial spin-labeling MR imaging in Moyamoya disease compared with clinical assessments and other MR imaging findings. Eur J Radiol 2013;82: e840-47 CrossRef Medline

15. Goetti R, O'Gorman R, Khan N, et al. Arterial spin labelling MRI for assessment of cerebral perfusion in children with Moyamoya disease: comparison with dynamic susceptibility contrast MRI. Neuroradiology 2013;55:639-47 CrossRef Medline

16. Wang R, Yu S, Alger JR, et al. Multi-delay arterial spin labeling perfusion MRI in Moyamoya disease: comparison with CT perfusion imaging. Eur Radiol 2014;24:1135-44 CrossRef Medline 